\title{
The Day, the Month, and the Year: What Plato Expects from Astronomy
}

\author{
István M. Bodnár
}

\begin{abstract}
The Timaeus apparently assigns a different task to astronomy than that in the educational programme set out in the Republic. There is no word about the reorientation required in the Republic that astronomers should ascend to a post-observational study of "the real decorations [of the heavens] - the real movements that these move by true quickness and true slowness in true number and in all true figures in relation to each other, carrying along the things contained in them, which can be grasped by reason and thought, and not by sight." (Republic 529d) Nevertheless, I argue that - albeit with vastly different theoretical presuppositions about perceptible entities - the Timaeus takes into consideration some of the strictures of the Republic. Similar to the way the reform of astronomy required in the Republic, only such observational astronomy can pass muster in the Timaeus whose major aim is to reduce the regularities of the motions of the different celestial objects to components that are connected to the fundamental motions of the World Soul. This enterprise can be claimed - within the confines of this likely story- to integrate in its fully developed form every important intellectual pursuit there is.
\end{abstract}

\section{Keywords}

Plato - Timaeus - observational astronomy - educational programme - celestial periods - component motions - intellect - unity of science

If we compare the account of astronomy in the educational programme of the Republic and in the Timaeus, we see that in the Timaeus astronomy has taken on a central role it was not yet assigned in the Republic. That much should be small wonder: if you are harangued by someone who is a most accomplished astronomer, and has made the greatest effort to know about the nature of the universe (27a), ${ }^{1}$

1 The formulation of Timaeus 27a might be taken to suggest that this is true only in compari-

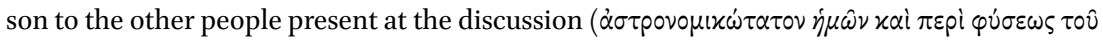

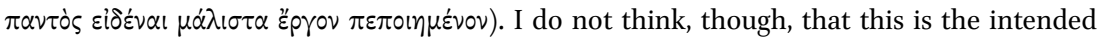


you surely should expect a very favourable, or indeed, a biased task assignment with respect to astronomy.

Call this the deflationary reading. Here I will argue that there is more to the new task assignment in the Timaeus than a mere marketing ploy on behalf of someone whose views are not endorsed by Plato. Indeed, why would Plato canvass these views, unless he intends to give them at least a favourable hearing, to the extent a likely story deserves? But once the views about the role of astronomy expressed by Timaeus are admitted to bear on Plato's own thought, an assessment of the differences between the Timaeus and the Republic is in order.

This comparison should address two different, but intricately related issues. One, the revision of the place of astronomy in a framework of intellectual enlightenment, the other the content of astronomy. Both of these problems should be subsumed under the broader question of the status of these different frameworks of intellectual enlightenment. This last issue I will not address in detail. Nevertheless, on occasion, I will have to remark that the different assessments of astronomy are maintained in two different modalities: one in the straightforward exposition of the philosophically grounded pedagogical reform of the Republic, the other as part of the likely story of the Timaeus.

1

First, the Republic. A full discussion of the role of the mathematical disciplines in the educational programme of the Republic would exceed the limits of a paper. Here I can give only very brief assertions, with just the bare minimum of a sketch of the argumentation for my proposals. For a comparison with the Timaeus at least three major issues should be broached. First, presumptions about the status of celestial entities, which bear upon what can, and what cannot, be achieved by astronomy. Second, the reform of astronomy Socrates proposes in the face of these limitations. And finally, third, the place of astronomy within the curriculum.

Celestial entities are, on Socrates' telling, in principle of the same kind as everyday objects around us, even if their behaviour follows very regular patterns.

point of Critias' remark: Timaeus is an accomplished astronomer in his own right and the comparison is made in order to explain why Timaeus - and not someone else from among those present-will be speaking in the main part of the dialogue. This hypothesis is also borne out by Socrates' characterisation of Timaeus at the beginning of the dialogue, that "he has come to occupy positions of supreme authority and honor in his city [and] has, in my judgment, mastered the entire field of philosophy" (20a-translations from the Timaeus, unless otherwise indicated, are from Zeyl's translation in Cooper, ed. Plato: Complete Works). 
But as for the ratio of night to day, of days to a month, of a month to a year, or of the motions of the stars to any of them or to each other, don't you think [the real astronomer will] consider it strange to believe that they're always the same and never deviate anywhere at all or to try in any sort of way to grasp the truth about them, since they're connected to body and visible?

$530 \mathrm{a}-\mathrm{b}^{2}$

This is in full agreement with Socrates' earlier injunction that "we should use the decoration in the sky as models (paradeigmasi) in the study" of the real objects of astronomy (529d, Grube-Reeve translation, slightly modified). These are:

the real decorations - the real movements that these move by true quickness and true slowness in true number and in all true figures in relation to each other, and they carry along the things contained in them which can be grasped by reason and thought, and not by sight.

$529 \mathrm{~d}$, my translation

This last phrase, to the effect that these true motions (or the true decorations performing these true motions) carry along the things contained in them, is crucial, because it makes clear that even though there is an ontological divide between the true objects of astronomy and what is observable in the heavens, the true objects of astronomy have a similar structure to the objects observable in the heavens and their visible motions. This is indeed why the latter can be used as models on the basis of which the truly insightful and informative astronomical "problems" (53ob) can be formulated, and then solved, even when in the course of this investigations we can "leave the things in the sky alone" (53ob). ${ }^{3}$

This means, then, a thorough revamping of astronomy as Plato's contemporaries knew it. In the first, interrupted introduction of astronomy, Glaucon submitted that astronomy provides knowledge of seasons, months and years (527d), indispensable for agriculture and for military purposes alike. This position is, however, rebuffed by Socrates, who insists that the mathematical

2 Translations from the Republic, unless otherwise indicated, are from the Grube's translation revised by Reeve in Cooper, ed., Plato: Complete Works.

3 This characterisation is paralleled by the way Socrates chastises the harmonic theory of the Pythagoreans, who "seek out the numbers that are to be found in these audible consonances, but they do not make the ascent to problems." (531c) For further discussion of Plato's criticism of Pythagorean harmonic theory, see Huffman, Archytas of Tarentum: Pythagorean, Philosopher and Mathematician King, 423-425. 
studies should not be oriented towards practical ends. Instead they should lead the soul to grasp reality, that is, they should further the philosophical, or dialectical cognition which follows the mathematical part of the curriculum $(527 \mathrm{~d})$. Then, again, as I have quoted above, Socrates submits that "as for the ratio of night to day, of days to a month, of a month to a year, of the motions of the stars to any of them, or to each other [...]" it is strange "to try in any sort of way to grasp the truth" about the perceptible heavenly bodies, or about the perceptible periods of these bodies (530a). If the usual astronomical method of observation and geometrical modelling cannot provide certainty about these motions, the task must be reformulated as one of grasping those real, not-perceptible movements which true astronomy aims at uncovering and understanding. But grasping these real movements will not mean that through them we will be able to account for the residual irregularity of the motions of these celestial entities. Such irregularity is inherent in their bodily nature.

Socrates does not give an example of what the inquiry into these nonperceptible movements should be like. Nevertheless, the context-astronomy is introduced after stereometry-strongly suggests that we should expect a geometrical analysis of complex motions into component revolutions. But even if the contents of this astronomy remain underspecified, the place of astronomy is clearly set out in this educational programme. The would-be guardians start with arithmetic. This is then followed by geometry-two-dimensional geometry, that is. Then, Socrates remarks that the lack of a developed stereometry should be remedied, and only after this can astronomy be tackled. After astronomy, harmonics completes the curriculum. Astronomy holds a central place in this programme - it comes after stereometry, but it also relies on arithmetic, because it investigates:

the real movements, which they [the true decorations] move by true quickness and true slowness in true number and in all true figures in relation to each other.

$529 d$, my emphasis

The next step, harmonics, a mathematical investigation of the audible feature of motion, does not immediately follow arithmetic. In contrast to astronomy, the investigation of motions for which the eyes were framed, harmonics is the investigation of motion for which the ears were framed. ${ }^{4}$ These sciences are,

4 Here I follow Shorey's translation of pepēgen and pagènai of 53 od (Shorey, The Republic, 189). In note $d$, Shorey convincingly compares the use of this verb here to the use at 6o5a. Another consideration in favour of this understanding of the verb is that on this interpretation the 
accordingly, sort of sister sciences (adelphai tines), as Socrates asserts, referring approvingly to the Pythagoreans. If this relationship is closer than those between all the other mathematical sciences, this might motivate us to place them together. ${ }^{5}$

We do not know, however, why these sister disciplines are given in the order astronomy-harmonics, and there is not much point in speculating about this order. We should, nevertheless, note that the order of the two sister sciences reverses the order arithmetic-geometry, and, even more importantly, astronomy is not presented as the culmination of mathematical education. This is even clearer, if we keep in mind that the list of mathematical disciplines is in an important sense open-ended in the Republic. Socrates warns at $530 \mathrm{ff}$. that the Pythagoreans must be consulted about the characterisation of the five sciences on the curriculum, and asked whether they have any other mathematical disciplines to add. Socrates does not indicate that he would rule out the inclusion of these further Pythagorean candidates in the educational programme. ${ }^{6}$ Instead, he insists that the would-be guardians:

passage would be in line with Timaeus' assertions about sight, namely that the observation of the heavens is the "supremely beneficial function for which the god gave [the eyes] to us"

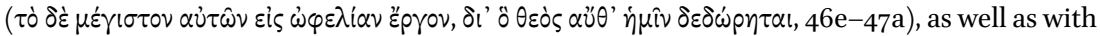
the assertion about sound and hearing that "[l]ikewise, the same account goes for sound and hearing - these too are the gods' gifts, given for the same purpose and intended to achieve

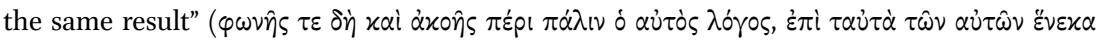
$\pi \alpha \rho \dot{\alpha} \theta \varepsilon \omega \hat{\omega} \nu \delta \varepsilon \delta \omega \rho \hat{\eta} \sigma \theta \alpha \mathrm{l}, 47 \mathrm{c}-\mathrm{e})$. The other option is to follow the Grube-Reeve translation, then eyes and ears "fasten on" (= "are attentively directed to") these two kinds of motions.

5 The investigation of mathematical studies should aim at bringing out their community (koinōnia) and kinship (syngeneia), and those respects in which they are akin ( $\tau \alpha \hat{\tau} \tau \alpha \hat{\eta} \hat{~ \varepsilon ̇ \sigma \tau i \nu ~}$

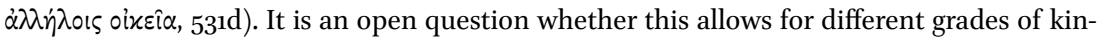
ship among the different mathematical sciences. Cf. Archytas B1, where all four branches of mathematics - astronomy, geometry, arithmetic and harmonics - are said to be sister sciences (or kindred sciences, the term used is adelphea).

6 Here I deviate from Burnyeat's interpretation in Burnyeat, "Plato on why mathematics is good for the soul," 16-19. Note that I concur with Burnyeat's formulation that "[a]ny science that does not lend itself to such redirection [i.e. redirection to the same abstract level as geometry or arithmetic] is to be excluded altogether"(18). I differ, however, in maintaining that Socrates does not pass judgment on whether such a redirection is possible in the case of those sciences which will be established during Plato's lifetime. Accordingly, I do not think that Plato blacklisted mathematical mechanics and optics (cf. Burnyeat, "Mathematics," $16 \mathrm{f}$.). What he must insist on in their case is the same as what he did in the case of Pythagorean astronomy or harmonics: they do not have to be blacklisted. Instead they can be admitted to the programme after proper reorientation.

Note that beyond the reorientation of astronomy and harmonics there is the further reform of arithmetic and geometry, although in their case this does not extend to changing their standard operational procedures. In the case of astronomy and harmonics the reform is not just one of placing the discipline in a larger epistemological and ontological scheme 
should never try to learn anything incomplete of these, anything that doesn't reach the end that everything should reach — the end we mentioned just now in the case of astronomy.

53 oe

The last requirement, I take it, stipulates that these new candidates for the educational programme should be reformed in the same way astronomy has been: whatever the primary manifestations are that they study, they have to acknowledge that these do not constitute the actual objects of their investigation. Instead they should concentrate on those true-that is, non-perceptible and immutable - realities which form their proper object of study. Indeed, immediately after these admonitions the next discipline on the programme, harmonics, will undergo exactly this same kind of reform at the hands of Socrates.

But if there are further possible candidates lined up for admission to the programme, the relative place of astronomy within this programme is even less secure. The objectives and the results of astronomy remain the same, but astronomy might turn out to have a slightly different contribution to the interdependence of mathematical disciplines depending on what exactly these disciplines turn out to be.

2

In turning to the Timaeus we can follow the same checklist we covered in the Republic. First, we need to check what presumptions are operative about the status of celestial entities and celestial motions. Next, we will need to discuss whether Timaeus' practices amount to something like a reform of astronomy as prescribed by the Republic. And finally, we will turn to the question I raised in my title: what does Plato expect from astronomy in the Timaeus?

All of the celestial entities-planets and fixed stars alike-are visible and generated gods (4od). ${ }^{7}$ Among these, the case of the planets (the Sun and the Moon included) is complicated, as Timaeus admits at $38 \mathrm{~d}-\mathrm{e}$ and $4 \mathrm{Oc}-\mathrm{d}$. The fixed stars are gods, whose bodies are made mostly out of fire (40a). These follow the motion of the circle of the Same, revolving around the Earth, and have an additional rotation of their own. The important difference from the account

(Burnyeat, "Mathematics," 42). In their case a major reassessment and change of the standard aims and procedures of the disciplines are also in order.

7 For further discussion of these corporeal gods see Broadie, "Corporeal Gods, with Reference to Plato and Aristotle," and Betegh's contribution in this volume. 
of the Republic is that the revolution these entities perform under the causal influence of the circle of the Same does not admit of variations. No matter that these stars are corporeal, perceptible entities, they "stay fixed by revolving without variation in the same place" within these celestial revolutions (4ob). Similarly with the planets. Timaeus does not go into details, nevertheless he submits that Venus and Mercury, have the same period as the Sun, but that Mercury and Venus receive a contrary power to that of the Sun. This does not mean that they have a revolution in a contrary sense to that of the Sun. Instead the claim is that "the Sun, the star of Hermes [i.e. Mercury] and the Dawnbearer [i.e. Venus] overtake one another and are overtaken by one another in the same pattern" (38d, Zeyl translation, slightly modified). ${ }^{8}$ Timaeus does not specify the details of how this celestial pas de trois is accomplished. Nevertheless, it is clear that the fundamental claim, namely that the period of these planets is identical, is valid, and will always remain valid. Similarly, for the other planets:

the wandering of these bodies [is] bewilderingly numerous as they are and astonishingly variegated[, i]t is none the less possible, however, to discern that the perfect number of time brings to completion the perfect year at that moment when the relative speeds of all eight periods have been completed together [...].

$39 \mathrm{c}-\mathrm{d}$

Again, the situation is the same as in the case of Sun, Mercury and Venus: the very complex motions these planets may perform do not rule out in the least that they have constant periods, and that these periods together give rise to an overarching regularity like the Great Year. Again, this presupposes that behind all the variation and complexity some periodicities of planetary motion are, and will always remain, valid.

This claim, that celestial revolutions are endowed with some fundamental constancy makes sense in the context of the Timaeus in light of two further considerations. One is that the eternity and constancy of features of celestial objects is intimately related to what the Demiurge solemnly announces about the products of his making. These entities, "as creatures that have come to be, are neither completely immortal nor exempt from being undone. Still [they] will not be undone [...] since [they] have received the guarantee of [the Demiurge's] will" (41b). This guarantee, as the Demiurge hastens to add, "is

8 I translate kata tauta "in the same pattern." The phrase may express nothing more than that, when any of these three planets overtakes another one among them, the latter must as a next step overtake the former. 
greater [...] than those with which [these entities] were bound when [they] came to be." These entities, then, possess some greater, divinely ordained stability than what their constitution, on its own, could ever provide them with.

Moreover, the divine guarantee of the eternity of created corporeal entities also rests on the fact that celestial bodies, and the body of the whole cosmos as such, are under the causal influence-or even: the guidance and careof soul. In the case of celestial bodies, the soul is directly responsible for the motion of these bodies, that is why Timaeus speaks about setting the fixed stars "in the wisdom of the dominant circle" (40a), whereas the planets are set "into the orbits traced by the revolution of the Different" (38c, Zeyl translation, slightly modified) - the Moon in the first circle, the Sun in the second and so on with respect to the other planets. This means that the motions of the celestial bodies have more regularity than their material constitution would allow for; their motion is dependent on the operation of the World Soul.

All of these psychological and divine warrants, then, allow that - contrary to the claims of the Republic - observational astronomy can be pursued in a meaningful way. Note that this may still allow for discrepancies between the supremely, unerringly regular motion of the World Soul, ${ }^{9}$ which is evidenced in the sky, and the actual corresponding revolution of a planet. Nevertheless, even in such cases the discrepancies cannot accumulate: if the path of a planet for any reason does not match the revolutions of the World Soul, such a discrepancy cannot be augmented to the point when the planet would be out of sync with whatever overall regularity the revolution of the World Soul has. And come to think of it, this is already a lot to ask by the lights of the Republic. ${ }^{10}$

But this observational astronomy, as I shall argue, takes into consideration some of the strictures of the Republic. This is possible, because where the Timaeus and the Republic differ, they do so within the context of their vastly different theoretical presuppositions about the investigation of perceptible

9 See $47 \mathrm{~b}-\mathrm{c}$, speaking about the function of sight "that we might observe the orbits of intelligence in the universe," in the course of which we will realise that these "universal orbits are undisturbed" and as a result we can "imitat[e] the completely unstraying revolutions of the god."

10 Note that the characterisation I have given here about planetary motions has obvious resemblances to the description of planetary motions in the Myth of Er, in Book x of the Republic. There the motions of the celestial bodies are induced by the Moirai, each of them providing different component motions-Clotho the diurnal motion of the sky, Atropos provides contrary motions for each of the planets, whereas Lachesis provides additional motions in both directions, deflecting the planets from their course provided by Atropos, and then directing them back again to where they should have been, and beyond (see $617 \mathrm{c}-\mathrm{d})$. On the planetary account of Republic x see Knorr, "Plato and Eudoxus on the Planetary Motions." 
entities. Most importantly, the more positive account of the Timaeus is set out within the framing of an account which is introduced as a likely story. So whatever positive assurances Timaeus gives us about the stability of the world and the feasibility of investigating it, this remains embedded in the larger claim that what he sets out is only a likely story. So the change between the two dialogues is not so much one of fundamental convictions, but rather an appreciation of how the fundamental divide between the non-perceptible, immutable realm and the physical realm does nevertheless allow for a meaningful mathematical investigation of some parts of the physical, an investigation which at the same time will appeal to the teleological considerations, reaching into psychology and theology. In a way, then, the Timaeus can be looked upon as a dialogue which after the critical attitude of the Republic provided the necessary theoretical assurances for a feasible research programme in astronomy.

Not of any old kind of astronomy though. As I have already indicated-and I will come back to it shortly - this astronomy is one which does relate celestial motions to some non-perceptible, non-physical motion similar to the way the reform of astronomy required in the Republic. This is intimately connected to a further restriction: those cosmological accounts which deploy theories of retardation are ruled out. This is clear from the characterisation of the planetary periods:

Some [planets] would move [with their own movement, of the Different] in a larger circle, others in a smaller one, the latter moving more quickly and the former more slowly. Indeed, because of the movement of the Same, the ones that go around most quickly appeared to be overtaken by those going more slowly, even though in fact they were overtaking them. $39 \mathrm{a}^{11}$

It is only in such composite motions as the one Timaeus propounds that it is meaningful to raise problems about the issue of which of the two celestial

11 Cf. Laws 822a-b: "This belief, my dear fellows, that the moon and sun and other stars 'wander' in any way whatsoever, is incorrect: precisely the opposite is true. Actually, each of them covers the same path, and not many, but always a single one in a circle, although it is true that to all appearances it moves many paths. Further, the quickest body is wrongly supposed to be the slowest, and the opposite [i.e. the slowest—is wrongly supposed] to

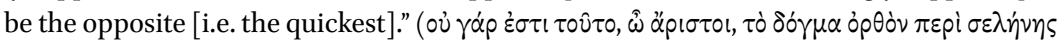

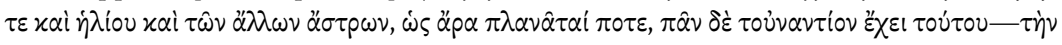

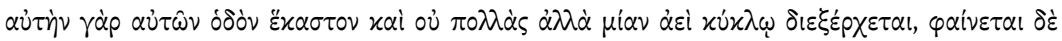

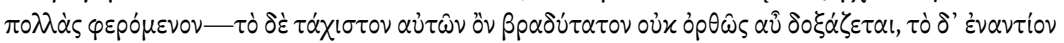

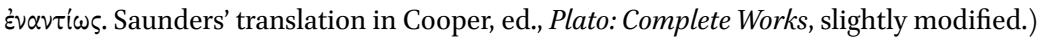


bodies overtakes the other one. In models of retardation-or in vortex models, as they are usually called—such issues are completely straightforward. Whichever celestial body performs a quicker overall revolution, will be the one that overtakes the celestial body with a slower overall period. ${ }^{12}$

In contrast to such a model, we can be certain that in Plato's case the rejection of the retardation model means that the motion of the apparently slower celestial body must be parsed in terms of at least two component motionsone, which is identical for all of the celestial bodies, and another, in an opposite sense, which is responsible for the differences between the motion of the circle of the fixed stars and those of the different planets, and between the motions of the different planets themselves. I do not think much argumentation is needed to show that this kind of analysis into component motions adheres to the injunctions of the Republic, namely that celestial motions should be treated as problems which invite general considerations and solutions. Such a methodological precept should not rule out, even by the lights of the Republic, that the astronomer takes his cue from the celestial motions themselves.

This is brought into sharp relief if we compare the strictures of the Republic with the account of how the Demiurge kindles light in the circle of the Sun with the aim of:

bestow[ing] upon all those living things appropriately endowed and taught by the revolution of the Same and the uniform, a share in number. $39 \mathrm{~b}-\mathrm{c}$

Then, as instances of the numbers inculcated by the Same and the uniform under the illuminating light of the Sun, the day, the month and the year are mentioned: periods, that is, whose precise investigation was branded as futile by the Republic.

Note, however, that the Timaeus passage does not just bypass the strictures of the Republic. It immediately conveys a theoretical understanding of these periods, over and above any observational accuracy there may be in their case. Most importantly, as the story of the kindling of the light in the circle of the Sun suggests, all three periods rely fundamentally on the presence of the Sun. Most

12 Such vortex theories as Timaeus rejects were widespread: Anaxagoras and Democritus subscribed to some version of such a theory, see e.g. Anaxagoras A $78=$ Aëtius II 16.1

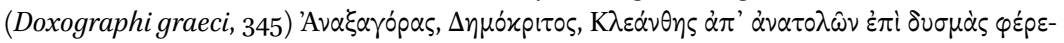

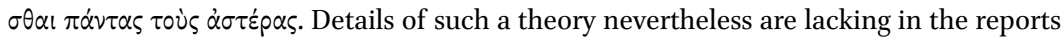
about Anaxagoras and Democritus. 
straightforwardly, "a year [comes to be],13 when the Sun makes a turn of its own circle" (39c, my translation), as Timaeus puts it. Also unmistakable is the role of the Sun in the case of the month: "A month [comes to be] when the Moon having made a turn of its own circle overtakes the Sun" (39c, my translation).

This neat connexion between the basic periods and the motion of the Sun may seem to break down in the case of day and night. Jowett, Archer-Hind, Cornford and Zeyl (and I trust, countless other modern translators) apparently presume that day and night correspond directly to the revolution of the Same, and the only role of the Sun in this case is that it accentuates this revolution by illuminating half of the Earth by its daylight. Of all these many translations it is sufficient to quote the one by Cornford:

Thus and for these reasons day and night came into being, the period of the single and most intelligent revolution.

$39 \mathrm{c}^{14}$

This, however, would be bad astronomy - as, for instance, was already objected by Taylor: the period of the circle of the Same, the sidereal day, is slightly shorter than the mean solar day. Moreover, it would make what Timaeus says terribly awkward: night and day may well be generated by kindling the light of the Sun on the second orbit, but the revolution of the circle of the Same-or the period of this revolution, as some translators put it-exists irrespective of whether the light of the Sun was kindled. Neither the revolution, nor its period is brought into being by the Demiurge's intervention when he kindled the Sun on an orbit around the Earth.

13 I translate the verb gegonen in the present tense, because although Timaeus formulates his claim in present perfect, he does not speak about the historical generation of the first day, or first month, or first year. This is clear from the clauses about the month and the year. The conditional clauses are formulated with epeidan plus conjunctive and hopotan plus conjunctive constructions respectively, expressing the recurrence of the astronomical events which give rise to the periods, of a month and of a year, respectively. Accordingly, the sentence with its perfect tense main verb stresses that these periods have been established through the kindling of the light of the Sun to be recurrent phenomena. (Cf. Cornford, Plato's Cosmology, 115, which uses the present tense "comes to be"supplied from the context-for these latter two clauses, whereas Cornford translates the first, and actually only occurrence of the verb gegonen in the past tense as "came into being.")

14 Cornford, Plato's Cosmology, 115. See furthermore Jowett, The Dialogues of Plato, 458 (cf. also Jowett's paraphrase: "The revolution of the world around the earth, which is accomplished in a single day and night, is described as the most perfect or intelligent." 404), Archer-Hind, The Timaeus of Plato, 129, and also Zeyl's translation. 
One alternative solution to this conundrum is to bite the bullet and suggest that Timaeus' list of the generation of night and day, of the month and of the year deliberately starts with a curious oversimplification, or-if you wishwith a riddle. Whereas in the case of the month and the year, Timaeus provides the correct account, the first instance, that of day and night, is presented with the problematic identification of the two periods. As for the emergence of "the period of the single and most intelligent revolution" itself, perhaps one way of evading the difficulty could be to suggest that the presence of the light of the Sun dramatically accentuates the change caused by this revolution. Without the sequence of days and nights the revolution would not be noticeable. As an additional consideration for this one could adduce that according to $39 \mathrm{e}-4 \mathrm{ob}$ the fixed stars are "set into the thought of the most powerful [circle], following it" (40a, my translation) only after the coming to be of time. Accordingly, at this point the motion of the Same is not yet articulated by these fixed stars.

But it is one thing to imply the prior unnoticeability of "the period of the single and most intelligent revolution," and another to imply its prior absence. Hence it may be preferable to take the sentence in a different construal. Here Timaeus certainly speaks about the generation of night and day, but he need not be understood as speaking about the generation of the revolution of the circle of the Same. Instead, he can call attention to the fact that once the Sun lights up in the sky, night and day come to be, ${ }^{15}$ and the revolution of the circle of the Same comes to be night and day. And the change of this revolution into night and day can acknowledge the difference between the length of the revolution itself, which is the sidereal day, and the length of the solar day. ${ }^{16}$

15 The coming to be of night and day, of month and of year was already announced earlier: "For there were no days and nights, no months, and no years before the heavens came to be. But now, at the same time as the heavens are organized, he devises their coming to be" (37e, Zeyl's translation, slightly modified).

Note furthermore that the coming to be of night and day by kindling light in the orbit of the Sun also presupposes the presence of the Earth, the "guardian and maker of night

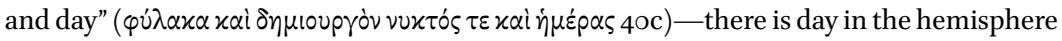
lit by the Sun, whereas in the other hemisphere there is night.

16 Another construal of the clause to the same effect could be to delete the comma before $\dot{\eta}$

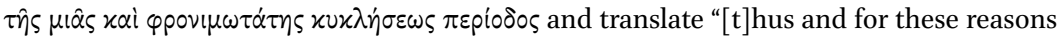
the period of the single and most intelligent revolution came to be night and day." This then would be followed up by the two further claims "and it comes to be a month when the Moon having made a turn of its own circle overtakes the Sun, and a year when the Sun makes a turn of its own circle" (39c, my translation). This reading, however, could be problematic on two counts. First of all, readers of the Timaeus have been primed by the announcement of $37 \mathrm{e}$ to expect the coming to be of days, nights, months and years, and not something coming to be them. Moreover, even though the revolution of the circle 
Such an understanding fits smoothly with the context: the immediately following clause- "a month comes to be when the Moon, having completed its own cycle, catches up with the Sun"17 — describes how the period of the Moon relates to the synodic month. Indeed, the relationship between the two different days is somewhat similar to that between the two different months: in the time that it takes for the orbit of the Moon to make a complete revolution, the Sun has covered almost 1/13 part of its annual orbit. In order to reach alignment, the Moon, after completing its own circuit, will also need to catch up with the much slower Sun. In the case of the day, the circle of the Same and that of the Sun revolve in opposite directions, but the Sun also takes part in the revolution of the Same. ${ }^{18}$ Consequently, between e.g. two middays the circle of the Same needs to make more than one revolution, since the circle of the Sun has covered almost $1 / 366$ part of its own orbit in the meantime. The circle of the Same, accordingly, has to cover additional distance, and this takes some additional time - on account of which the solar day, between e.g. two middays, is slightly longer than the sidereal day of a single revolution of the circle of the Same. Or, put differently: once night and day are determined by the presence (and absence) of the light of the Sun, having a slow contrary motion to the revolution of the circle of the Same, the cycle of night and day will not be exactly the original period of the circle of the Same. Instead, just as Timaeus puts it, through the presence of light in the circle of the Sun "night and day come to be, the turning of the single and most intelligent revolution [comes to be night and day]," indicating not only that this revolution will be perceived differently by the presence of light, but also allowing for the slight difference between the two periods - that of that most intelligent revolution, the sidereal day, and that of the period of day and night, the solar day. ${ }^{19}$

It is instructive to contrast this understanding of the passage with Taylor's remarks on $39 \mathrm{c} 2 \mathrm{ff}$. at this point. In exact opposition to my interpretation above, he bases his understanding of the text on the claim that "the distinction between the mean solar day and the sidereal day [...] was not yet discovered"

of the Same can be looked upon as coming to be night and day, it is only a sequence of several such revolutions that can come to be a month, or a year.

1739 c, my translation.

18 In the account of the difference between the synodic and sidereal month we did not need to take into account this additional component, because both Sun and Moon take part in the diurnal revolution of the stars.

19 By speaking about the solar day without further specification I intend to avoid using any more precise indication, like the apparent solar day, or the mean solar day. Timaeus speaks about a single "orbit traced by the revolution of the Different" for each planet, for considerations about the possible discrepancies between these revolutions and the actual path of the planets see p. 119, at n. 10 above. 
and draws the immediate conclusion that "[t]he period [i.e. of the complete revolution of the circle of the fixed stars] is therefore taken to be 24 hours, a nychthēmeron." ${ }^{20}$

This, as I have suggested above, is already problematic. ${ }^{21}$ But it is further compounded by Taylor's claims about the month: "the month is said to run from one conjunction of moon and sun to the next, and at the same time to be the period of one revolution of the moon in its orbit." Most importantly, it is gratuitous to claim that Timaeus would have asserted that these two periods are the same. Instead, what Timaeus says is that "[a] month [comes to be] when the Moon having made a turn of its own circle overtakes the Sun," and this allows for the Moon first completing its own cycle, and then overtaking the Sun only afterwards. All in all, Taylor's closing remark that "As no figures are given, we may perhaps fairly suppose that Timaeus is not distinguishing either of these 'months' from the conventional 'calendar month'" is unwarranted. There are ways of distinguishing the sidereal and the synodic months without

Taylor, A Commentary on Plato's Timaeus, 214.

This was first pointed out by Vlastos, Plato's Universe, Appendix C, 100:

There is a problem here which Cornford and most other commentators have ignored. Plato appears to be talking as though the period of the diurnal revolution of the sun - the solar day - were identical with the movement of the Same, while his theory requires it to be a little shorter [...]. Only A. E. Taylor [...] shows awareness of the difficulty, and he cuts the knot by declaring that "the distinction between the mean solar day and the sidereal day [...] was not yet discovered" paying no attention to the fact I have just mentioned: that on the very theory expounded in the Timaeus there must be a small, but appreciable, difference between the two units.

Vlastos, nevertheless, keeps the traditional translation, claiming that "Plato is overlooking (and not denying) the difference [between the two periods] in this context" (ibid.). He supports this claim by two considerations. Firstly, that the teleological function of making humans aware of number, is served by the "dramatic alterations of day and night" (101). This is correct—and the clause indeed mentions night and day — but the passage does not require Plato's overlooking the difference, just as there is no need to suppose (as Taylor does, see my critical remarks in the main text above) that the following lines require overlooking the difference between the sidereal and the synodic month. As a second consideration Vlastos remarks that "for practical, calendric, purposes the solar day is inevitably the basic unit of measurement," and Greek astronomers use this unit in their calendar reforms by devising different Great Years (ibid.). Nevertheless when Timaeus introduces his own perfect year that is completed "when the relative speeds of all eight periods have been completed together and, measured by the circle of the Same that moves uniformly, have achieved their consummation" $\left(39 \mathrm{~d}_{4}-7\right)$, the reference to the period of the motion of the Same among the eight periods mentioned need not refer to solar days. Indeed, the question - apart from the issue of contention, namely the interpretation of $39 \mathrm{c}$-will be moot: if there is a Great Year of exactly $k$ solar years comprising of altogether exactly $n$ solar days, that period will be exactly $n+k$ sidereal days long, as each year is exactly one day longer measured in sidereal days than it is in solar days. 
providing specific values for the length of either of them. Indeed, the way in which Timaeus describes the month may make it clear beyond reasonable doubt that he is speaking about the synodic month, taking it to be longer than the specific period of the circle of the Moon, i.e. the sidereal month.

Accordingly, Timaeus' description indicates that what we encounter in celestial cycles are actually complex phenomena and not just the different periods of the revolution of the circle of the Same, or of the different circles of the Different. This is so in the case of the month, and can well be so in the case of night and day. And even though the period of the orbit traced by the revolution of the Different where the Sun is set is identical to the year, neither is this period given to us in complete isolation. The motion of the Same was given dominance over the motions of the Different ( $36 \mathrm{c} 7 \mathrm{f}$.). In order to grasp the motions of the Different one needs to isolate them from the overbearing influence of the motion of the Same. In the case of the Sun, across the year the daily path of the Sun will be somewhat different from day to day. It would be quite an obvious move to attribute the period of days and nights to the motion of the Sun. Timaeus instead stressed that this period is at its root the revolution of the Same (perhaps combined with the motion of the Sun), under the influence of the light of the Sun. When it comes to identifying the period of the orbit of the Sun one needs to grasp the component motions of its complex motion: the overbearing daily component, of the revolution of the Same, and the other revolution with a yearly period. Indeed, reverse-engineering the period of the yearly motion of the Sun, and the possible reverse-engineering of the period of the day and night into the two component motions should run on a parallel track. These are also a major prerequisite for a similar analysis of the motions behind the period of the synodic month.

As a result, Timaeus' astronomy will turn out to be observational astronomy, but its major aim is not just to record the regularities of the motions of the different celestial objects. Instead, the aim of this astronomy is to grasp the fundamental motions of the World Soul which give rise to the periods and regularities that can be detected in these complex motions.

This, then, can serve as a cue for us to return to our initial question-what did Plato expect from astronomy in the Timaeus? As we have seen, in an important way Timaeus' astronomy is an exercise in reverse-engineering celestial motions onto the workings of the universal soul. At this point we should look 
at this process of reverse engineering in some more detail. For a start, Timaeus submits that perceiving the astronomical regularities evokes a rudimentary grasp of number $(47 \mathrm{a}-\mathrm{c})$. Once number is invented and time is understood-I trust in terms I have just set out-the nature of the universe can be investigated, and philosophy can be worked out.

For all of the obvious differences, this line of development has, in various ways, some affinity with the educational curriculum of the Republic. Number plays a crucial role in both accounts, even though its origin is characterised differently: according to Timaeus it is astronomical regularities which induce us to count, whereas in the Republic just about anything can start us off in the direction of numbers: in the example starting at $523 \mathrm{c}$, Socrates illustrates the problems arithmetic tackles by counting up three fingers.

After this initial triggering of the recognition of number, celestial motions continue to aid further studies - astronomy prominent among them-and lead to a full scale investigation into the nature of the universe. This may be thought to include a somewhat smaller amount of mathematics than what featured on the curriculum of the Republic, but that will depend on what is contained in the investigation about nature, and in the whole realm of philosophy. Philosophy here is the greatest gift from the gods to the human race-I take it, it is the summit of intellectual insight, comparable in status to the dialectics of the Republic. This does not, however, settle the question of its content yet. Timaeus' remarks could still allow that after the triggering and initializing functions of pre-astronomy and astronomy the two latter phases of the investigation into the nature of the universe, and of philosophy are decoupled from what instigated their occurrence first, and afterwards they constitute separate disciplines in their own right. Nevertheless, the phrasing of $47 \mathrm{a}-\mathrm{b}$ (speaking about "the realm of philosophy"), and also the passage, where Socrates introduces Timaeus at 200 as someone who has mastered the entire field of philosophy, ${ }^{22}$ suggest that the realm of philosophy can stand for a collection of different, but related intellectual pursuits, and these can also encompass the mathematical disciplines. ${ }^{23}$

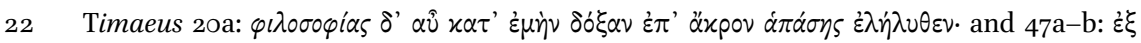

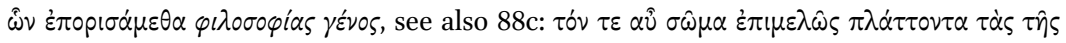

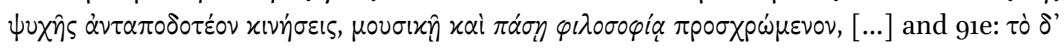

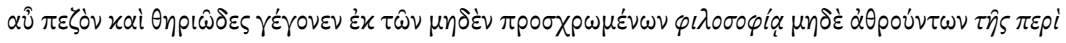

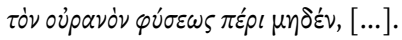

23 Cf. also the question Socrates asks from Theodorus at the beginning of the Theaetetus:

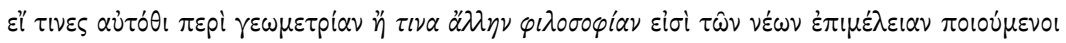
(143d). 
Hence, what is truly remarkable about the Timaeus most probably does not lie in restricting the importance of mathematical knowledge as compared to the Republic. Instead, the Timaeus account differs markedly from the educational programme of the Republic in that it stresses the seamless integration of philosophical insight with mathematical, and within that, with astronomical understanding. The most important factor in this integration is the description how the World Soul, a supreme subject of cognition uses its circles, of the Same and of the Different, in order to grasp in an adequate manner the different objects of cognition. In this model, whatever counted as mathematical or dialectical cognition in the Republic will be performed by the circle of the Same $(37 \mathrm{a}-\mathrm{c})$. Once, however, this supreme subject of cognition, which is to some extent accessible to our senses, is admitted in the Timaeus, the aim and objective of human cognition can be described in two related ways: one is that we have to complete the entirety of the realm of philosophy (as Timaeus puts it at $47 \mathrm{a}-\mathrm{b}$ ), and the other, that we have to grasp and imitate the operations of this huge celestial mind: we have "to observe the orbits of intelligence in the universe and apply them to the revolutions of our own understanding," as a result we will "come to know them and [...] share in the ability to make correct calculations according to nature," this will "stabilize the straying revolutions within ourselves by imitating the completely unstraying revolutions of the god." $(47 \mathrm{~b}-\mathrm{c})^{24}$

To put it otherwise, the process of human development can be described both in terms of the objects of cognition, and in terms of the subject of cognition, and Timaeus' claim is that due to this latter type of description astronomy, or one might even say, an astronomically backed psychological theory, is a crucially important asset to mend our own souls, damaged on delivery $(47 \mathrm{a}-\mathrm{c}$ and gob-d). Accordingly, the right kind of astronomy is not just another mathematical discipline among many, but it is the foremost discipline to understand and imitate the operations of the World Soul. Hence it will be intimately connected to and, indeed, in its fully developed form it will in a sense integrate every important intellectual pursuit there is.

This would be a huge claim, or even an incredible one about any science, even discounting the possibility that Timaeus might be overselling his likely

24 Note, that this is true only about astronomy practiced in the appropriate way. Indeed, the stakes can be menacingly high in this respect: people engaged in astronomy that is not pursued in the right way- "innocent but simpleminded men, who studied the heavenly bodies but in their naiveté believed that the most reliable proofs concerning them could be based upon visual observation" - will be deprived of their humanity on rebirth and will have to lead their next life as birds of the sky (91d-e). 
story about the creation of the cosmos. But there is a more charitable way of setting out these large claims of the Timaeus. They may be taken as propounding that the knowledge we gain about the world cannot be neatly parcelled out into separate disciplines. When someone is out to understand the operations of the celestial mind, that should not just be astronomy: it should include a full grasp of how this world came about, what sort of paradigm the Demiurge used, what sort of considerations he had, and further, a full grasp of the connexions between the paradigm and whatever other paradigms there are included in it. Add furthermore all the things the World Soul may be thinking about-an imitation of these revolutions may very well require that to the extent it is humanly possible, we should entertain the same thoughts as the World Soul. Both of these considerations, then, suggest that on the Timaeus' likely story, the understanding we should seek about celestial revolutions should not stop at the mathematical level of investigation. ${ }^{25}$

\section{Works Cited}

Archer-Hind, R. D. The Timaeus of Plato. London: Macmillan and Co., 1888.

Broadie, Sarah. "Corporeal Gods, with Reference to Plato and Aristotle." In $\Sigma \Omega M A$ : Körperkonzepte und körperliche Existenz in der antiken Philosophie und Literatur. Edited by Thomas Buchheim, David Meissner and Nora Wachsmann. Hamburg: Felix Meiner Verlag, 2016.

Burnyeat, Myles. "Plato on why mathematics is good for the soul." Proceedings of the British Academy 103 (2000): 1-81.

Cooper, John and David S. Hutchinson, eds. Plato: Complete Works. Indianapolis: Hackett, 1997 .

Cornford, Francis. Plato's Cosmology: The Timaeus of Plato. London: Routledge, 1935.

Huffman, Carl. Archytas of Tarentum: Pythagorean, Philosopher and Mathematician King. Cambridge: Cambridge University Press, 2005.

Jowett, Benjamin. The Dialogues of Plato. Vol. III. 3rd ed. Oxford: Clarendon Press, 1892. Knorr, Wilbur R. "Plato and Eudoxus on the Planetary Motions." Journal for the History of Astronomy 21 (1990): 313-329.

25 I am grateful to the participants of the Prague meeting for questions and critical remarks. I was also greatly helped by written comments from Gábor Betegh, and also by his forthcoming paper "Cosmic and Human Cognition in the Timaeus." The final revision of the paper was supported by a sabbatical leave from Eötvös University, a faculty fellowship at the Institute for Advanced Study of Central European University, and by the project ОткA K-112253. 
Shorey, Paul. Plato: The Republic. With an English translation, vol. ii. London: William Heinemann - Cambridge, Mass.: Harvard University Press, 1935.

Taylor, A. E. A Commentary on Plato's Timaeus. Oxford: Clarendon Press, 1928.

Vlastos, Gregory. Plato's Universe, Seattle: University of Washington Press, 1975. Reprinted with a new introduction by Luc Brisson. Las Vegas: Parmenides Publishers, 2005. 